\title{
A Circularly Polarized Antenna Array with a Convenient Bandwidth/Size Ratio Based on Non-Identical Disc Elements
}

\author{
Stefano Maddio*
}

\begin{abstract}
This paper presents the design and development of a circularly polarized microstrip antenna array for C-band application. The proposed antenna has a convenient trade-off between bandwidth and dimension. The array design is based on the sequential phase arrangement (SPA) of $2 \times 2$ non-identical disc-based patch elements, operating in modal degeneration. Exploiting the properties of the SPA, capable of forcing $\mathrm{CP}$ even when operating on linear polarized elements, each disc is independently detuned to operate on non-perfectly overlapping bands. When properly fed by a sequential phase network (SPN), the set of four discs seamlessly covers the wide cumulative bandwidth which is the combination of the four sub-channels. To verify the design, a single-layer via-less array was fabricated in a compact printed square board of side $40 \mathrm{~mm}$, meaning a surface of $0.64 \lambda_{0}^{2}$ at the center frequency of $6.0 \mathrm{GHz}$, assembling the elements with a compact space-filling SPN. Measurements show a wide $10 \mathrm{~dB}$ return loss bandwidth of $28.5 \%$, a $3 \mathrm{~dB}$ axial ratio (AR) bandwidth exceeding $1 \mathrm{GHz}$, and a realized gain ranging from $4.1 \mathrm{~dB}$ to $7.25 \mathrm{~dB}$ inside the $\mathrm{AR}$ bandwidth. The global bandwidth of the proposed array, almost coincident with the AR bandwidth, is $17.0 \%$.
\end{abstract}

\section{INTRODUCTION}

In modern communications and sensor applications, printed antennas are preferred for their advantageous cost - benefit trade-off. The diffusion of wideband applications has pushed the limits of the microstrip antenna design, trying to enlarge its inherent narrow band nature. This is particularly challenging for the class of antennas operating in circular polarization (CP), exploiting the modal degeneration principle [1, pp. 496]. Sequential phase arrangement (SPA) is a diffuse approach to enlarge the bandwidth of CP antennas [1, pp. 499,2]. The rationale of SPA is the sequential rotation of the array elements with the excitation of sequential phase. The actual rotation angle and incremental phase $\Delta \phi$ depend on the number of elements. The most diffuse implementation is based on four elements arranged in a $2 \times 2$ square lattice fed with $90^{\circ}$ of $\Delta \phi$.

While the bandwidth of arrays based on the SPA principle is unmistakably enlarged compared to the bandwidth of the elementary antenna, it nonetheless still depends on it. To overcome this limit, in this paper the resonant frequency of each element is slightly detuned, leading to a set of antennas operating on non-coincident bands, covering a wider frequency spectrum when considered as a superposition. When the elements are fed by a properly designed SPN, the entire cumulative band is seamlessly covered by the sequential activation of each resonance, provided that the individual frequency bands overlap enough to avoid interruption. This also holds for the asymmetry issues of the pattern, which are mitigated by a moderate frequency detuning.

Following this principle, a CP antenna array with enhanced bandwidth for applications in the $C$ band has been designed and fabricated. The set of $2 \times 2$ non-identical disc-based elements is fed with an ultra-compact SPN arranged on a square printed board built with single-layer via-less technology. The device, fabricated with standard photo-etching technique on a high quality substrate, exhibits more

Received 17 August 2015, Accepted 4 October 2015, Scheduled 19 October 2015

* Corresponding author: Stefano Maddio (stefano.maddio@unifi.it).

The author is with the Department of Information Engineering, University of Florence, V.S. Marta, 3, I-50139, Florence, Italy. 
than $1 \mathrm{GHz}$ of $3 \mathrm{~dB}$ axial ratio (AR) bandwidth, meaning $17.6 \%$ of fractional bandwidth, a peak gain of $7.25 \mathrm{~dB}$ with a $3 \mathrm{~dB}$ gain tolerance bandwidth of $1.2 \mathrm{GHz}$, and a $10 \mathrm{~dB}$ return loss (RL) bandwidth exceeding $28 \%$. The effective bandwidth, almost coincident with the AR bandwith, is $17.0 \%$ with a normalized area of $0.64 \lambda_{0}^{2}$ at $6.0 \mathrm{GHz}$.

\section{ARRAY DESIGN}

\subsection{Analysis of the Elements}

Figure 1 shows the basic geometry of the elementary antenna and its characteristics. It consists of a single feed circularly polarized disc patch, already presented by the author [3-5]. A brief summary of its characteristics will be given here. The geometry of the patch is determined by fixing the external radius $R$, the axes of the ellipse, labeled by $A$ and $B$, and the angle $\theta$ individuated by the feeding line with the ellipse reference - cf. Figure 1(a). The disc operates in modal degeneration, with the ellipse serving as the degeneration driver, splitting the fundamental $\mathrm{TM}_{11}$ mode of the disc into two detuned ones, $\mathrm{TM}_{11}$ and $\mathrm{TM}_{y}$, due to the differently forced current paths. Qualitatively, the modes behave as $\mathrm{TM}_{11}$ of the unperturbed disk, radiating linearly polarized (LP) orthogonal far-fields, as depicted in Figure 1(b) and Figure 1(c). With the the disc radius fixed, the actual modal frequencies depend on the dimensions of the ellipse, while the angle $\theta$ acts as a mixing parameter for the two modes. This behavior is well illustrated by the circuital equivalence approach.

In Figure 2, the edge-referred input impedance for a generic design is depicted as $\theta$ varies from $0^{\circ}$

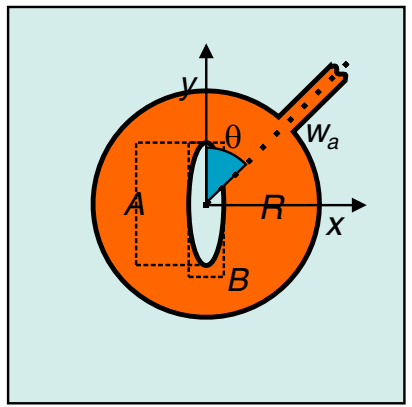

(a)

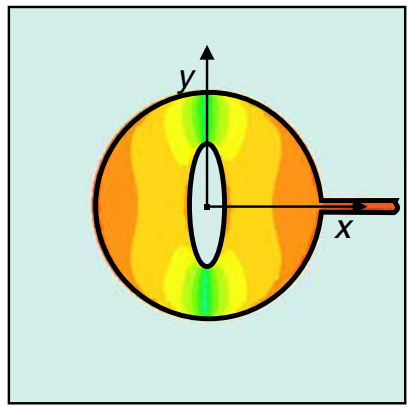

(b)

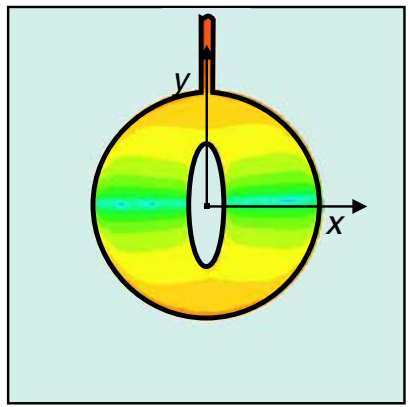

(c)

Figure 1. Disc geometry and pictorial representation. (a) Disc Geometry. (b) Mode $\mathrm{TM}_{x}$. (c) Mode $\mathrm{TM}_{y}$.

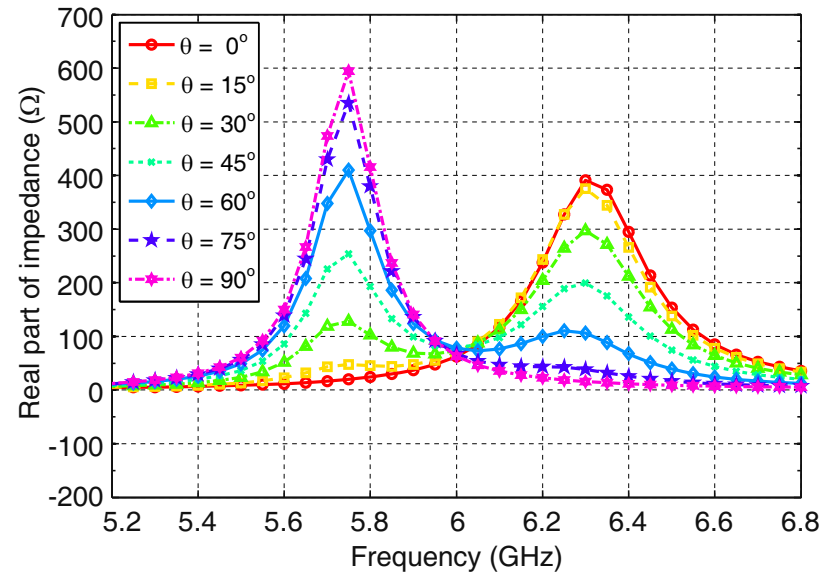

(a)

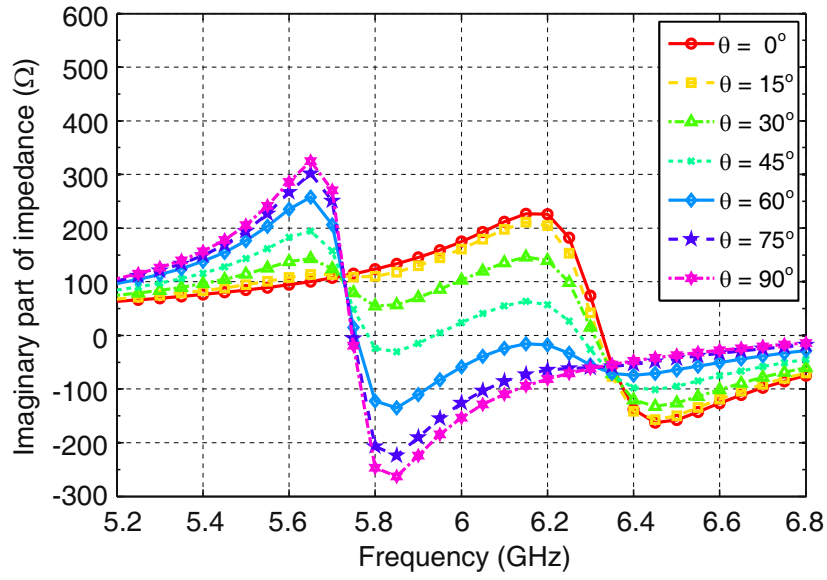

(b)

Figure 2. Input impedance $Z_{i n}(f)=Z_{x}(f)+Z_{y}(f)$ as $\theta$ is varied from $0^{\circ}$ to $90^{\circ}$. 
to $90^{\circ}$. According to the cavity model [1, pp. 257-262], limiting the analysis to the frequency range of interest, the impedance can be decomposed into the sum of two second-order resonators, which represent the responses of the two modes:

$$
Z_{i n}(f)=Z_{x}(f)+Z_{y}(f)=\frac{R_{x}(\theta)}{1+j Q_{x}\left(\frac{f}{f_{x}}-\frac{f_{x}}{f}\right)}+\frac{R_{y}(\theta)}{1+j Q_{y}\left(\frac{f}{f_{y}}-\frac{f_{y}}{f}\right)}
$$

with the circuital parameters, $R_{x}, R_{y}, Q_{x}, Q_{y}$ and $f_{x}, f_{y}$, as functions of the patch geometry and substrate characteristics $[1,3,6,7]$. With reference to Figure 1 , when $\theta=0^{\circ}$, the modal resistance $R_{x}$ is zero while $R_{y}$ is at its maximum, meaning that the higher mode $\mathrm{TM}_{y}$ is exclusively excited, and a $y$-directed far-field is generated - Figure 1(c). In a similar manner, when $\theta=90^{\circ}$, the lower mode $\mathrm{TM}_{x}$, associated with an $x$-directed far-field, is the only far field component - Figure 1(b). Between these extremes, both modes are excited with a ratio dictated by the correspondent impedance responses, i.e., $Z_{x}(f) / Z_{y}(f)$. As for each quasi-symmetrical shape, such as the cornered square, the nominal CP is obtained when the modal far fields are combined with equal magnitudes in phase quadrature $[1,6]$, conditions which can be imposed by operating on the equivalent circuit parameters $[3,5,7]$.

Instead of investigating the conditions for the optimal CP at a single frequency, a wide-spectrum analysis of the resonances is carried out. Figure 3 summarizes this analysis as two families of curves obtained by varying the axes of the ellipse when the external radius is $R=5.0 \mathrm{~mm}$ - Figure $3(\mathrm{a})$ and $R=6.5 \mathrm{~mm}$ - Figure 3(b). Confirming a similar analysis in [5, Figure 2], both $f_{x}$ and $f_{y}$ are found to be inversely proportional to both $R$ and $A \times B$ as dictated by the canonical disc loop closed-form response $[1,368-371]$. It can be observed that the relation between the geometric parameters and the actual frequencies is quite regular, easily fitted to a polynomial expression.

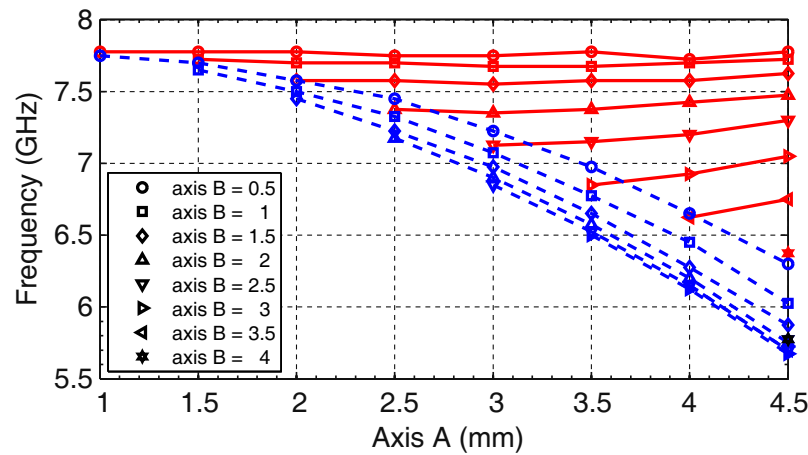

(a)

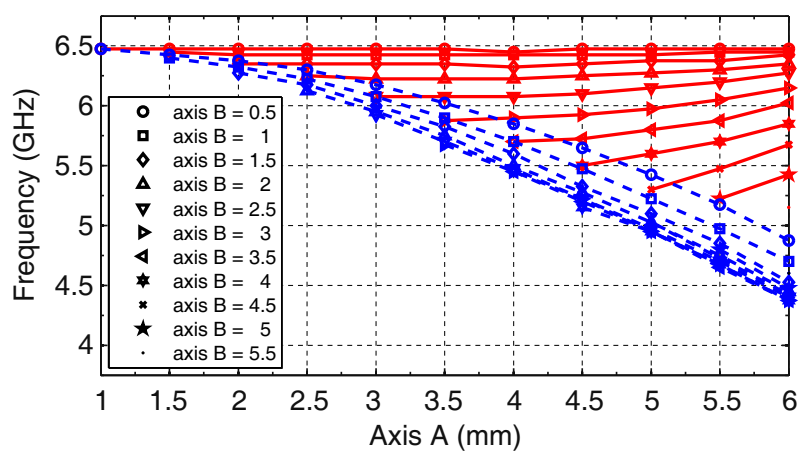

(b)

Figure 3. Modal chart when varying the ellipse. Continuous lines: higher modal frequency $f_{y}$, dotted lines: lower modal frequency $f_{x}$. (a) Modal chart for $R=5.5$. (b) Modal chart for $R=6.5$.

\subsection{Arrangement of the Elements}

On the basis of the previous modal analysis, the arrangement depicted in Figure 4(a) was the preliminary design. Four discs, characterized by different $R, A$ and $B$, are sequentially deployed with a geometric $90^{\circ}$ progression. Each disc is identified by a progressive number \# $N$, and its modal frequencies are labeled with $\# N x$ and $\# N y$, where the $x$ and $y$ labels are with respect to the local reference. The actual dimensions of the four discs are tuned to exhibit the frequency sequence $\# 1 x \# 2 x \# 3 x \# 4 x \# 1 y \# 2 y \# 3 y \# 4 y$. The sequence of frequencies is easily designed by exploiting the modal analysis in Figure 3.

Figure 4(b) depicts the simulated gain of each element as well as a pictorial stem series which symbolically describes the sequence of resonances. The angle $\theta$ of each element is independently tuned to excite the $x / y$ modal pair with almost the same magnitude in order to have an uninterrupted series of similar gain peaks, overlapping enough to be enforced by the sequential mechanism. It is worth remarking that each element $\# N$ is the source of a continuous radiation from an $x$-oriented LP at $f_{N x}$ to a $y$-oriented LP at $f_{N y}$ passing through a possibly imperfect CP at intermediate frequencies. 


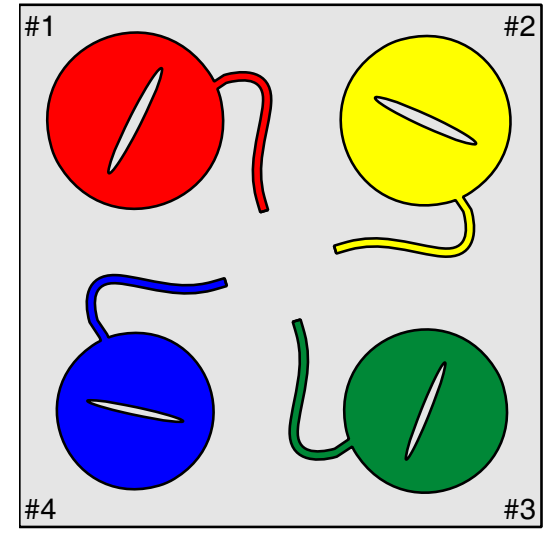

(a)

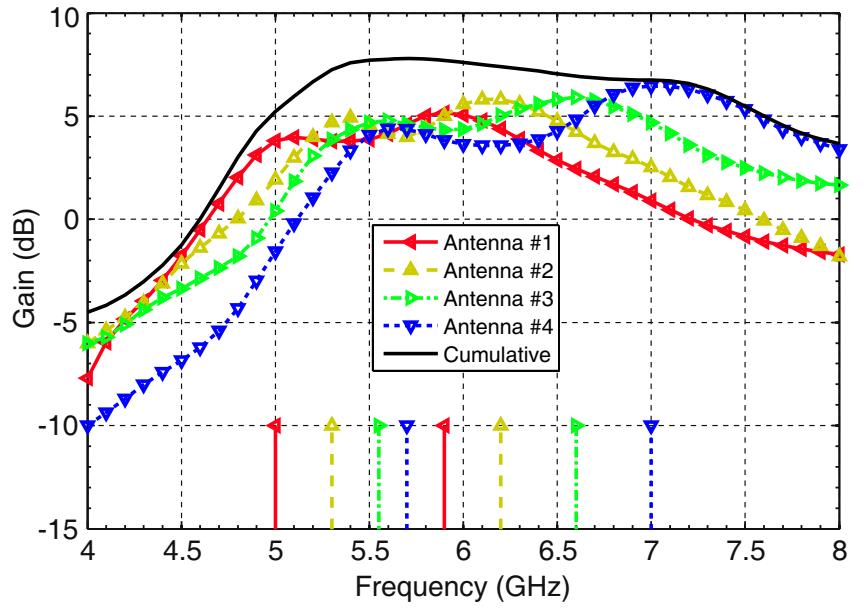

(b)

Figure 4. Layout and photograph of the proposed array. (a) Four differently tuned elements. (b) Individual and cumulative performance of the four elements.

The cumulative gain of the set, shown as the solid line in the same figure, is obtained by feeding all the elements with an ideal $90^{\circ}$ phase progression and equal magnitude, hence it is perfectly circularly polarized regardless of the polarization of each element. Albeit theoretical, this response demonstrates the feasibility of the proposed approach, at least in the asymptotic sense, resulting in the net enlargement of the cumulative bandwidth in comparison to the bandwidth of each antenna element taken by itself. The cumulative bandwidth can be made even wider if the elements' resonances are less overlapping. However, this would also affect the gain pattern, causing radiative inefficiency, pattern asymmetry, and matching problems.

\subsection{Assembling the Elements}

Figure 5(a) depicts the configuration of the typical sequential phase network (SPN) employed for the sequential arrangement of four antennas, seen as impedance terms $R_{L}$ [8-10]. Seven quarterwave transformers (QWT) are linked together, forming a sequence of four output branches, which are progressively rotated by $90^{\circ}$. The three internal QWT introduce the electrical phase rotation

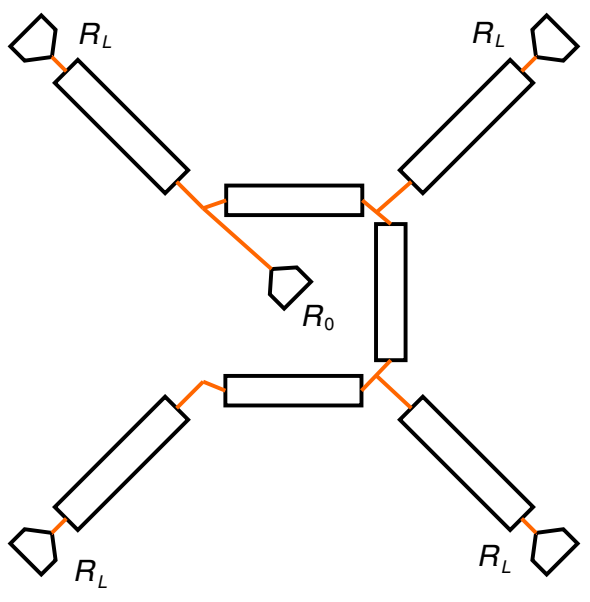

(a)

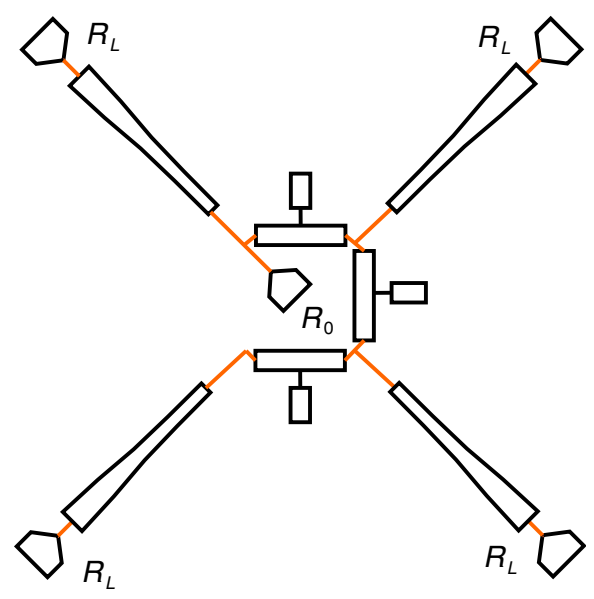

(b)

Figure 5. Equivalent circuits of the SPN. (a) Canonical architecture. (b) Modified architecture. 
of $90^{\circ}$ between each pair of elements, which compensates for the geometric rotation, resulting in an enhancement of the purity of the polarization.

To arrange the four non-identical discs in the limited space of $40 \mathrm{~mm} \times 40 \mathrm{~mm}$, the modified SPN design introduced by the author in [5], is applied here, hence briefly summarized. With reference to Figure 5(b), the three internal QWTs are replaced with stub-loaded T-section transmission lines. While providing the same electrical phase-shift of $90^{\circ}$, the T-stub sections are more compact and characterized by narrower line widths, an advantageous feature for the reduction of spurious radiation. Furthermore, the four output QWTs are replaced by tapered transmission lines to exploit the beneficial effects of wideband impedance matching. With reference to Figure 6(a), the tapered lines are curved to better accomplish the best space-filling arrangement, being longer than the nominal QWT. An additional stub is employed at the input section for fine-tuning the input impedance. Starting from the preliminary disc parameters giving the results in Figure 4(a), and from the nominal parameters for the network [10], a full-wave optimization has been carried out. The goal of the optimization is a Chebysheff-like behavior for both the realized gain and polarization purity over the largest possible bandwidth. The resulting design is depicted in Figure 6(a), and there is a detailed list of the geometric parameters in Table 1.

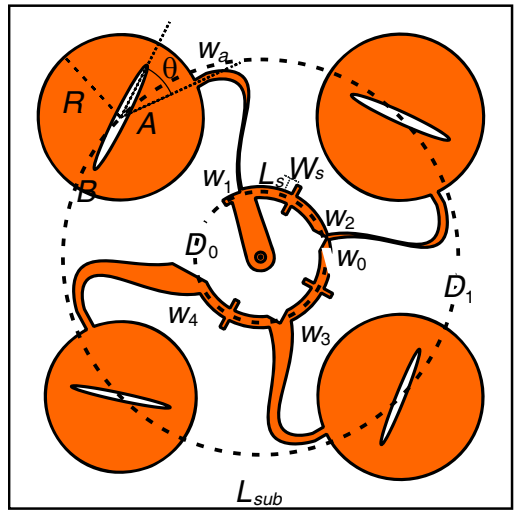

(a)

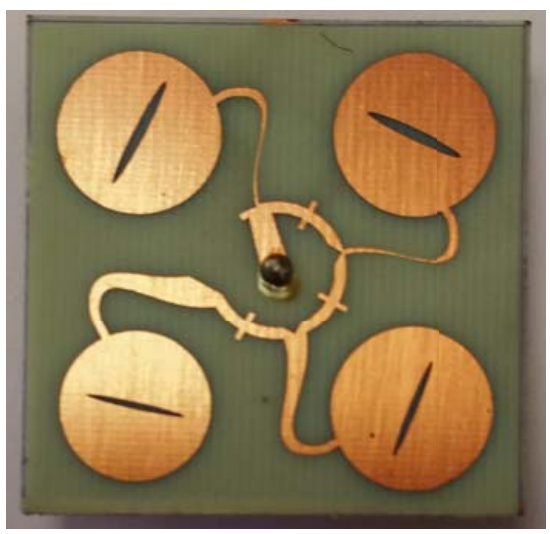

(b)

Figure 6. Layout and photograph of the proposed array. Dimensions are given in Table 1. (a) Design. (b) Photograph.

Table 1. List of the geometric parameters of the proposed array. The dimensions are in mm, the angles are in degree.

\begin{tabular}{|c|cccc|cccc|cccc|}
\hline Parameter name & $L_{\text {sub }}$ & $t_{\text {sub }}$ & $\epsilon_{\text {sub }}$ & $W_{a}$ & $W_{R}$ & $W_{0}$ & $W_{1}$ & $W_{2}$ & $W_{3}$ & $W_{4}$ & $W_{S}$ & $L_{S}$ \\
\hline Parameter value & 40 & 1.6 & 3.65 & 0.62 & 2.00 & 0.88 & 0.20 & 0.42 & 1.85 & 2.89 & 0.40 & 1.47 \\
\hline Parameter name & $D_{R}$ & $D_{0}$ & $D_{1}$ & $\theta_{\text {stub }}$ & $R_{1}$ & $A_{1}$ & $B_{1}$ & $\theta_{1}$ & $R_{2}$ & $A_{2}$ & $B_{2}$ & $\theta_{2}$ \\
\hline Parameter value & 2.20 & 5.17 & 15.72 & 40 & 6.57 & 4.63 & 0.47 & 40.4 & 6.62 & 4.08 & 0.46 & 42.5 \\
\hline Parameter name & $R_{3}$ & $A_{3}$ & $B_{3}$ & $\theta_{3}$ & $R_{4}$ & $A_{4}$ & $B_{4}$ & $\theta_{4}$ & & & & \\
\hline Parameter value & 6.57 & 4.01 & 0.34 & 45.2 & 6.00 & 4.00 & 0.30 & 55.0 & & & & \\
\hline
\end{tabular}

\section{EXPERIMENTAL VALIDATION}

A prototype of the proposed array, depicted in Figure 6(b), has been fabricated with standard photo-etching procedures on a high quality substrate (isola FR408: thickness $=1.6 \mathrm{~mm}, \epsilon_{R}=3.65$, $\tan \delta=0.001$ ), and then experimentally validated. In Figure 7(a), the measured and simulated reflection coefficients of the array are compared. The $10 \mathrm{~dB}$ RL defines a bandwidth from $5.2 \mathrm{GHz}$ to $6.9 \mathrm{GHz}$, i.e., a fractional bandwidth of $28.5 \%$. Despite a slight frequency shift, compatible with the fabrication tolerance, a good matching between the simulation and measurement is observed. 


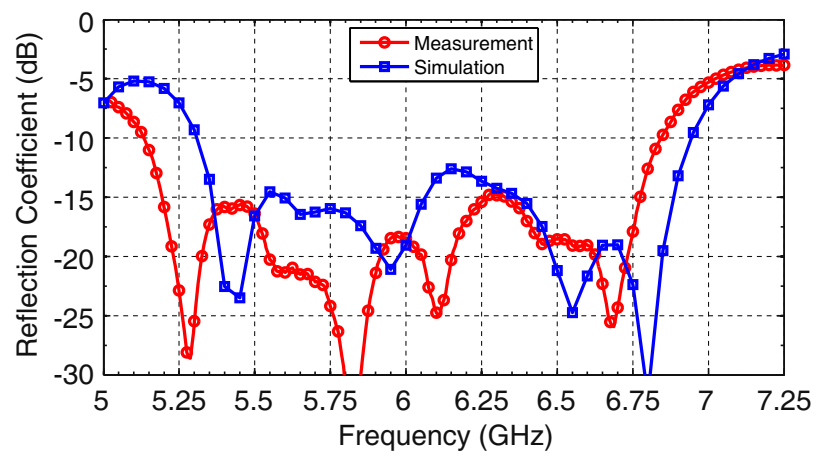

(a)

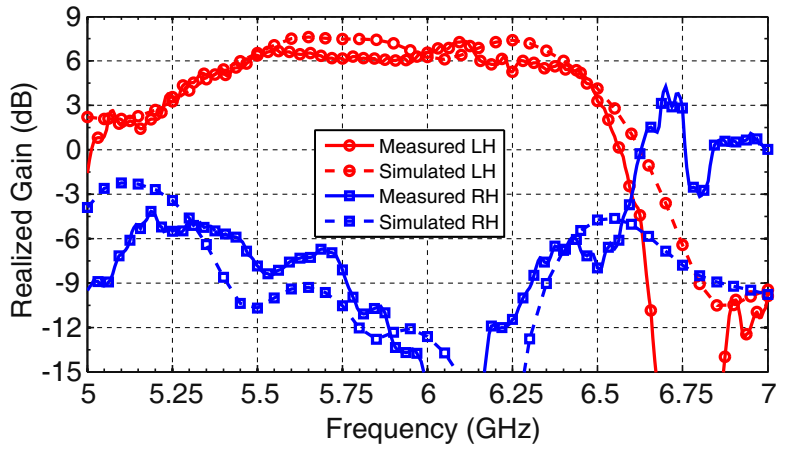

(b)

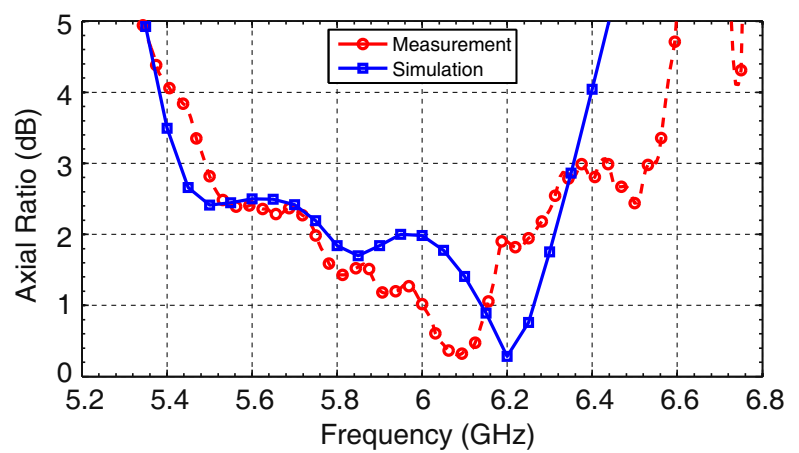

(c)

Figure 7. Layout and photograph of the proposed array. (a) Simulated and measured reflection coefficients. (b) Simulated and measured bore-sight gains. (c) Simulated and measured bore-sight axial ratio.

Table 2. A comparison of the proposed array with other similar arrays.

\begin{tabular}{|c|ccccc|}
\hline $2 \times 2$ Array & {$[11]$} & {$[12]$} & {$[10]$} & {$[5]$} & The present paper \\
\hline RL BW $(\mathrm{GHz})$ & $4.82-5.12$ & $4.46-5.5$ & $4.00-6.8$ & $5.00-6.75$ & $5.13-6.85$ \\
\hline AR BW $(\mathrm{GHz})$ & $4.84-5.13$ & $4.52-5.4$ & $5.10-7.0$ & $5.32-6.22$ & $5.49-6.55$ \\
\hline Gain BW $(\mathrm{GHz})$ & $4.86-5.12$ & $4.75-5.5$ & $5.12-7.0$ & $5.10-6.30$ & $5.35-6.51$ \\
\hline Global BW $(\%)$ & $5.2 \%$ & $13 \%$ & $20 \%$ & $15.5 \%$ & $17.0 \%$ \\
\hline Size $\left(\mathrm{cm}^{2}\right)$ & $100 \mathrm{~cm}^{2}$ & $81 \mathrm{~cm}^{2}$ & $81 \mathrm{~cm}^{2}$ & $20.25 \mathrm{~cm}^{2}$ & $16 \mathrm{~cm}^{2}$ \\
\hline Size $\left(\lambda_{0}^{2}\right)$ & $2.7 \lambda_{0}^{2}$ & $2.7 \lambda_{0}^{2}$ & $3.2 \lambda_{0}^{2}$ & $0.75 \lambda_{0}^{2}$ & $0.64 \lambda_{0}^{2}$ \\
\hline
\end{tabular}

The realized co-polar LH gain versus frequency is depicted in Figure 7(b), along with the crosspolar $\mathrm{RH}$ component. The gain response in the bore-sight direction is very flat, about $6 \mathrm{~dB}$ from 5.5 to $6.4 \mathrm{GHz}$, with a maximum value of $7.25 \mathrm{dBi}$ at $6.2 \mathrm{GHz}$. The limited value of the gain, compared to similar devices, is an inevitable consequence of the small area. The $3 \mathrm{~dB}$ gain tolerance bandwidth is quite in accord with the simulation results, extending from $5.25 \mathrm{GHz}$ to $6.4 \mathrm{GHz}$. The measured $3 \mathrm{~dB}$ AR bandwidth, depicted in Figure $7(\mathrm{c})$, spans from $5.49 \mathrm{GHz}$ to $6.55 \mathrm{GHz}$, i.e., $1.05 \mathrm{GHz}$ of left hand polarization bandwidth, corresponding to a fractional bandwidth of $17.6 \%$.

Figure 8 depicts the measured left hand pattern of the prototype for various frequencies. For each case, the $x$ - and $y$-cuts are given. Despite the lack of central symmetry, the pattern's symmetry is quite regular over the entire band of interest, with the exception of the extreme frequency of $6.5 \mathrm{GHz}$, which explains the behavior observed in the gain vs. frequency plot in Figure 7(b). As a side note, the frequency of $5.25 \mathrm{GHz}$, technically out of band, is still quite symmetric. 


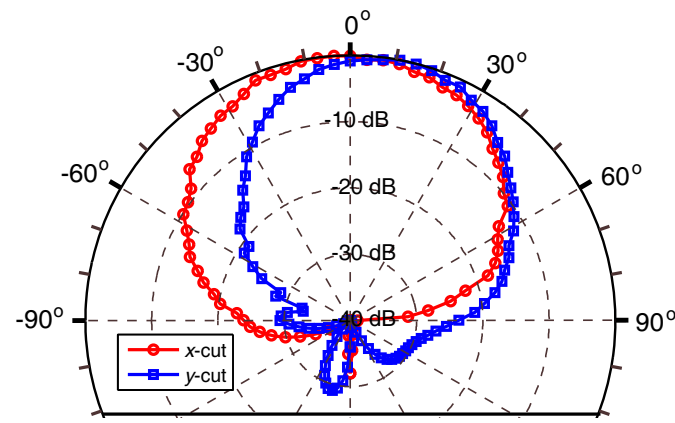

(a)

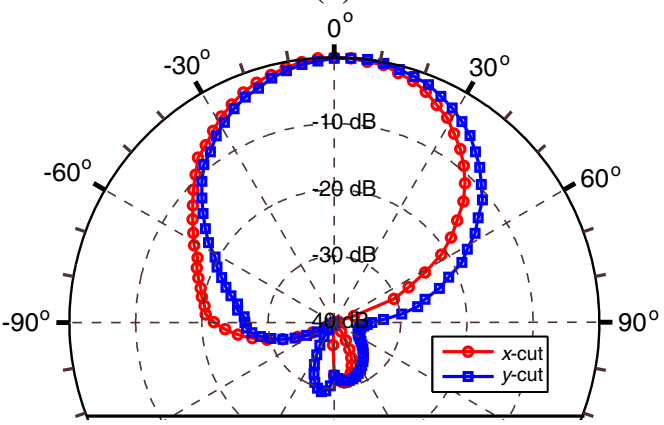

(c)

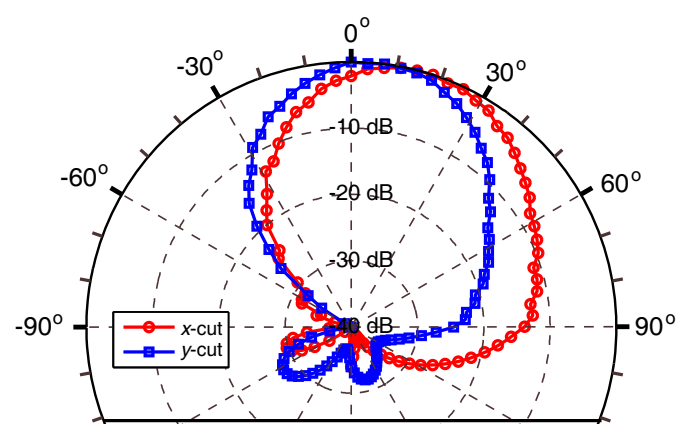

(e)

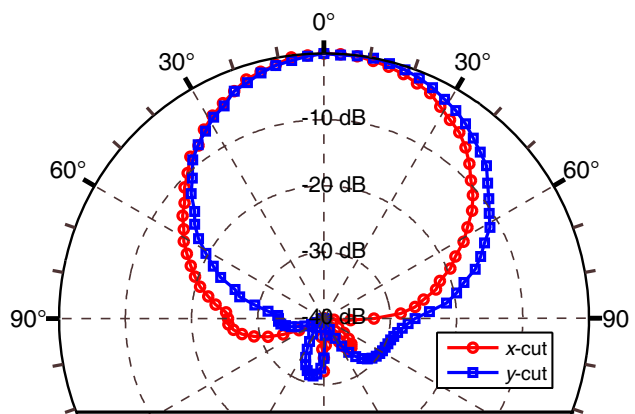

(b)

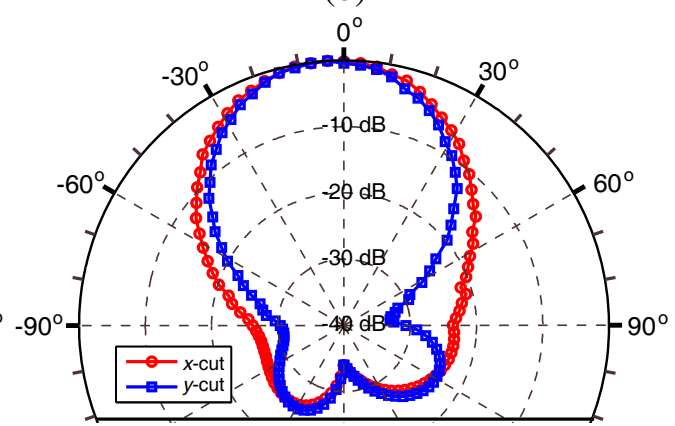

(d)

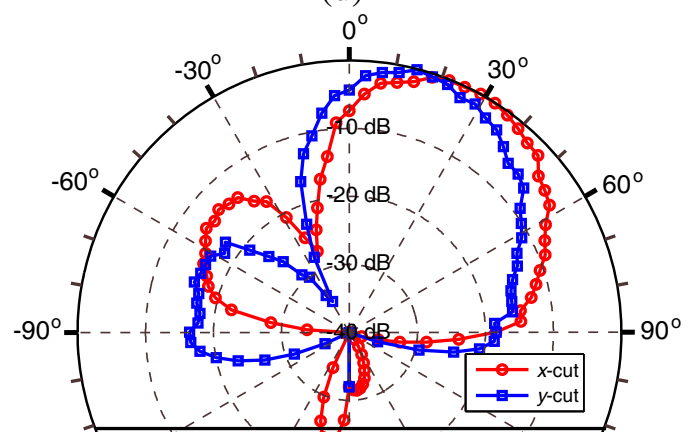

(f)

Figure 8. Principal cuts of the proposed array. (a) $5.25 \mathrm{GHz}$, (b) $5.50 \mathrm{GHz}$, (c) $5.75 \mathrm{GHz}$, (d) $6.00 \mathrm{GHz}$, (e) $6.25 \mathrm{GHz}$, (f) $6.50 \mathrm{GHz}$.

\section{CONCLUSIONS}

A compact circularly polarized antenna array for applications in the $C$-band was designed, simulated, and fabricated. The proposed array architecture is based on sequential phase arrangement applied to a set of 4 non identical disc elements. Arranged in a square of $40 \mathrm{~mm} \times 40 \mathrm{~mm}$, hence a surface of $0.64 \lambda_{0}^{2}$ at the mean frequency of $6.0 \mathrm{GHz}$, the fabricated device exhibits $28.5 \%$ of RL bandwidth, a peak gain of $7.25 \mathrm{~dB}$ with a $3 \mathrm{~dB}$ tolerance maintained over the range range $5.35-6.51 \mathrm{GHz}$, and a $3 \mathrm{~dB} \mathrm{AR}$ bandwidth spanning from $5.49 \mathrm{GHz}$ to $6.55 \mathrm{GHz}$, meaning $17.6 \%$. The global bandwidth of the device, defined as the intersection of the former bands, is $17.0 \%$, almost coincident with the AR bandwidth.

Table 2 shows a comparative summary of similar arrays found in the recent literature, demonstrating the validity of the proposed design as the one with the best bandwidth/dimension trade-off, at the sole price of a slightly reduced peak gain.

\section{REFERENCES}

1. Garg, R., Microstrip Antenna Design Handbook, Artech House, 2001. 
2. Hall, P., J. Huang, E. Rammos, and A. Roederer, "Gain of circularly polarised arrays composed of linearly polarised elements," Electronics Letters, Vol. 25, No. 2, 124-125, 1989.

3. Maddio, S., A. Cidronali, and G. Manes, "A new design method for single-feed circular polarization microstrip antenna with an arbitrary impedance matching condition," IEEE Transactions on Antennas and Propagation, Vol. 59, No. 2, 379-389, 2011.

4. Maddio, S., A. Cidronali, I. Magrini, and G. Manes, "A design method for single-feed wideband microstrip patch antenna for switchable circular polarization," IEEE European Microwave Conference, 262-265, 2007.

5. Maddio, S., "A compact wideband circularly polarized antenna array for C-band applications," IEEE Antennas and Wireless Propagation Letters, Vol. 14, 1081-1084, 2015.

6. James, J. R. and P. S. Hall, Handbook of Microstrip Antennas, IET, 1989.

7. Maddio, S., A. Cidronali, and G. Manes, "An azimuth of arrival detector based on a compact complementary antenna system," 2010 European Wireless Technology Conference, 249-252, 2010.

8. Evans, H., P. Gale, B. Aljibouri, E. G. Lim, E. Korolkwiwicz, and A. Sambell, "Application of simulated annealing to design of serial feed sequentially rotated $2 \times 2$ antenna array," IET Electronics Letters, Vol. 36, No. 24, 1987-1988, 2000.

9. Aljibouri, B., A. Sambell, and B. S. Sharif, "Application of genetic algorithm to design of sequentially rotated circularly polarised dual-feed microstrip patch antenna array," IET Electronics Letters, Vol. 44, No. 12, 708-709, 2000.

10. Rafii, V., J. Nourinia, C. H. Ghobadi, J. Nourinia, and B. S. Virdee, "Broadband circularly polarized slot antenna array using sequentially rotated technique for-band applications," IEEE Antennas and Wireless Propagation Letters, Vol. 12, 128-131, 2013.

11. Li, Y., Z. Zhang, and Z. Feng, "A sequential-phase feed using a circularly polarized shorted loop structure," IEEE Transactions on Antennas and Propagation, Vol. 61, No. 3, 1443-1447, 2013.

12. Yang, W., J. Zhou, Z. Yu, and L. Li, "Bandwidth and gain enhanced circularly polarized antenna array using sequential phase feed," IEEE Antennas and Wireless Propagation Letters, Vol. 13, No. 7, 1215-1218, 2014. 